\title{
The Density Spike in Cosmic-Ray-Modified Shocks: Formation, Evolution, and Instability
}

\author{
Byung-Il Jun and T.W. Jones \\ Department of Astronomy, University of Minnesota \\ 116 Church Street, S.E., Minneapolis, MN 55455
}

Received — 


\begin{abstract}
We examine the formation and evolution of the density enhancement (density spike) that appears downstream of strong, cosmic-ray-modified shocks. This feature results from temporary overcompression of the flow by the combined cosmic-ray shock precursor/gas subshock. Formation of the density spike is expected whenever shock modification by cosmic-ray pressure increases strongly. That occurence may be anticipated for newly generated strong shocks or for cosmic-ray-modified shocks encountering a region of higher external density, for example.

The predicted mass density within the spike increases with the shock Mach number and with shocks more dominated by cosmic-ray pressure. For very strong shocks, the total compression compared to the upstream gas may approach $\frac{\gamma_{g}+1}{\gamma_{g}-1} D$ during the formation period, where $\gamma_{g}$ is the gas adiabatic index and $D$ is the compression ratio through the precursor. As the full shock reaches equilibrium, the spike detaches, lags behind the modified shock transition and is further compressed, so that the density can exceed the limit quoted above. We find this spike to be linearly unstable under a modified Rayleigh-Taylor instability criterion at the early stage of its formation. Our linear analysis shows that the flow is unstable when the gradients of total pressure (gas pressure + cosmic-ray pressure) and gas density have opposite signs. We confirm this numerically using two independent codes based on the two-fluid model for cosmic-ray transport. These two-dimensional simulations show that the instability grows impulsively at early stages and then slows down as the gradients of total pressure and gas density decrease. Flow within the density spike becomes disordered through the instability. It seems likely that this can significantly increase the local magnetic field beyond compressional effects.
\end{abstract}


Observational discovery of this unstable density spike behind shocks, possibly through radio emission enhanced by the amplified magnetic fields would provide evidence for the existence of strongly cosmic-ray modified shock structures.

Subject headings: cosmic-rays - hydrodynamics — shock waves 


\section{Introduction}

Energetic charged particles (Cosmic-rays or CRs) are believed to be accelerated in strong shock waves through the first-order Fermi mechanism when they become trapped there by Alfvén wave turbulence. A relatively small population of particles can achieve very high energies by repeatedly crossing the shock and having their velocities redistributed by scattering. This very efficient, diffusive acceleration mechanism was discovered by a number of people independently in the late 1970s (Krymsky 1977, Axford et al., 1977, Bell 1978, Blandford \& Ostriker 1978). The process is nonlinear, since pressure feedback from accelerated CR particles modifies the shock compression and adjusts the acceleration efficiency towards an equilibrium (e.g., Blandford 1980). The modified shock structure includes an extended upstream pressure gradient ("foot" or "precursor") that results from upstream diffusion of CR pressure. Within the precursor gas is adiabatically compressed. At least during formation of the precursor there will also be a dissipative, gas subshock. However, above some critical Mach number diffusive shock theory suggests that this feature can become weak or absent (leading to a smooth or "C-shock" structure) in CR shocks as they approach dynamical equilibrium (e.g., Drury \& Falle 1986; Jones \& Kang 1990). The presence of a strong magnetic field transverse to the shock normal tends to reduce the

efficiency of CR acceleration in shocks of a given sonic Mach number (Webb et al. 1986, Jun et al., 1994, Frank et al. 1995), so the critical Mach number for the formation of a C-shock will generally be higher for MHD shocks (Jun et al., 1994).

The hydromagnetic structures of CR shocks are now fairly well studied for a variety of circumstances. Much of that effort has focussed on steady-state conditions (e.g., Drury \& Völk 1981; Webb et al. 1986; Ellison, Möbius \& Paschmann 1990; Kang \& Jones 1990; Jun et al., 1994). On the other hand, there are important circumstances in which steady-state CR shocks may not be applicable, such as supernova blast waves (e.g., Dorfi 
1984; Drury, Markewiecz \& Völk 1989; Jones \& Kang 1990). One interesting feature in time-dependent, evolving CR shocks is the appearance of a sharp density enhancement or "spike" downstream of the shock transition in both parallel and perpendicular shocks (Dorf) 1984; Dorfi 1985; Jones \& Kang 1990; Jun et al., 1994). This sharp density enhancement is formed as the growth of CR pressure first enhances the total compression through the shock and then levels off towards the equilibrium predicted for steady-state shocks. The maximum density within the spike can exceed that for the equilibrium modified shock and the initial shock by a large factor when the shock Mach number is large. We have discovered that this feature is Rayleigh-Taylor unstable. That could have interesting observable consequences, if, for example, it lead to amplification of magnetic fields within a well-defined region behind a supernova shock. In this paper, we present one- and two-dimensional numerical simulations of the evolution of the density spike, demonstrating its instability. We point out that the instability discussed here is distinct from other previously reported instabilities associated with the CR shock structure itself. On the other hand, the instability requires a large CR pressure and occurs because of modifications to the shock. Our simulations are based on the two-fluid formulation for CR transport (Drury \& Völk 1981). This method is much simpler than the more complete diffusion-convection formalism of CR transport, and it is still the only really practical method for studying multidimensional, time-dependent CR modified flows. Although the two-fluid method has limits (e.g., Jones \& Ellison 1991), recent work has demonstrated that the two-fluid formalism gives consistent solutions with diffusion-convection formalism in time dependent shocks when the necessary closure parameters are carefully defined (e.g., Kang et al., 1992; Frank et al. 1995; Kang \& Jones 1995; Kang \& Jones 1996).

The outline of this paper is as follows. The detailed description of the density spike formation is given in $\S 2$. In $\S 3$, we study the formation and evolution of the density spike in a various shock structures by means of one-dimensional numerical simulations. 
The modified criterion of Rayleigh-Taylor instability in the presence of CRs is discussed by solving the linear perturbation equations of the two-fluid system in $\S 4$. $\S 5$ presents our two-dimensional numerical results of the unstable density spike simulation, and we summarize in $\S 6$.

\section{Formation of the Density Spike}

Formation of the sharp density enhancement in CR modified shocks has been

explained previously (Drury 1987, Jones \& Kang 1990). We describe the process of density enhancement within CR-modified shocks more fully here in order to understand better what conditions may be relevant to the new instability we report. The sharp density enhancement or "spike" originates from development of or increases in the CR precursor. Diffusive acceleration at shocks depends on the ability of a fraction of high energy particles isotropized by scattering in the postshock flow to return upstream of the shock. Most of those particles are then advected back through the shock in a process that repeats. On balance these two effects confine the upstream CRs within a region whose scale is the diffusion length, $x_{d} \approx \kappa / v_{0}$, where $\kappa$ is a suitably defined mean spatial diffusion coefficient (see Drury \& Völk 1981), and $v_{0}$ is the upstream flow speed of the gas relative to the shock. If we define $x_{d}$ in terms of the cosmic ray pressure, $P_{c}$, and the CR pressure increase before the shock is $\delta P_{c}$, then there is a pressure gradient in front of the shock, $\sim \delta P_{c} / x_{d}$. That, in turn, decelerates the gas flowing into the shock, which produces an adiabatic compression and heating of the gas, adding to the total pressure gradient.

For strong, high Mach number and steady shocks this "precompression" and "preheating" can be sufficient to eliminate the need for a dissipative, gas subshock in order to satisfy the steady jump conditions for the shock (e.g., Drury \& Völk 1981; Malkov \&

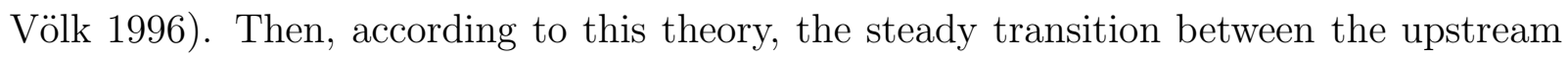


and downstream states will be continuous (a C-shock is produced). On the other hand such a shock will usually form from one that initially is not dominated by CR pressure. In other circumstances the Mach number of the shock may increase, for example, as the shock encounters a denser, colder environment or as the shock accelerates down a steep density gradient. That shock strengthening is followed by an analogous increase in the CR pressure and readjustment of the shock structure. In either of these two situations even an eventual C-shock will pass through an intermediate condition with a precursor and a gas subshock. In this transitional stage, the fluid is compressed twice; once by the precursor and once by the gas subshock. The precompression of the gas within the precursor automatically reduces the Mach number of the subshock. However, if the subshock is initially strong, then while the CR pressure at the shock is small compared to $\rho_{0} v_{0}^{2}$, the second density compression, through the subshock, is hardly reduced from its initial value. Consequently, the double transition of the flow can result in a density compression of the flow much more than the usual $\frac{\gamma_{g}+1}{\gamma_{g}-1}$ times the density far upstream, where $\gamma_{g}$ is the adiabatic index of the gas.

In order to determine approximately the maximum possible compression ratio without solving the full set of equations we can just use the above double compression concept. Let us define four distinct states in the flow for convenience. State "0" refers to flow conditions far upstream, ahead of the precursor. State "1" is that just upstream of the gas subshock, if it exists, while state " 2 " is that just downstream of the subshock. Then, finally "3" refers to the state that existed downstream of the subshock before the shock began adjusting to new upstream conditions. That region will propagate downstream, away from the subshock, and it is in the space between states " 2 " and "3" that the density spike feature develops. We also define the compression ratio through the precursor as $D \equiv \frac{\rho_{1}}{\rho_{0}}$. Recall that in this smooth transition the gas will be compressed adiabatically, so that $P_{g} \propto \rho^{\gamma_{g}}$. Then, one can use the gas adiabat and, assuming that the compression through the precursor is slowly changing, mass flux conservation to obtain the relation between the sonic Mach numbers 
$(\mathcal{M})$ in states " 0 " and " 1 ".

$$
\mathcal{M}_{1}=\frac{v_{1}}{c_{1}}=\frac{v_{0}}{c_{0}} \frac{1}{D^{\frac{\gamma_{g}+1}{2}}}=\frac{\mathcal{M}_{0}}{D^{\frac{\gamma_{g}+1}{2}}}
$$

where $c$ is the gas sound speed. Therefore, we can see that the sonic Mach number of the flow decreases as the flow experiences the adiabatic compression. If $P_{c 3}$ is less than the equilibrium value, then $P_{c 1}=P_{c 0}+\delta P_{c}$ will increase, causing $D$ to rise as well. The value of $D$ at equilibrium in a given shock depends on various details such as the Mach number of the shock, but, from conservation laws cannot exceed $D=D_{\max }=4$ if the net adiabatic index of the combined thermal/CR plasma is nonrelativistic or $D=D_{\max }=7$ if that plasma is relativistic. These limits would correspond to strong shocks that have been smoothed entirely into C-shocks by CR pressure (Drury \& Völk 1981).

As $D$ increases for a given $\mathcal{M}_{0}$ the value of $\mathcal{M}_{1}$ will decrease, down to a limit $\mathcal{M}_{1}=1$. If the entire steady-state jump conditions can be satisfied when $\mathcal{M}_{2} \geq 1$ the shock should evolve into a C-shock in which the downstream flow is supersonic (Drury \& Völk 1981). If the steady jump conditions across the full transition require a subsonic flow in region "2", then a subshock is required even as the shock reaches equilibrium. Applying the condition $\mathcal{M}_{1}=\mathcal{M}_{2} \geq 1$ for $D=D_{\max }$ leads to a minimum constraint on the Mach number $\mathcal{M}_{0}$ for a CR shock to be able to evolve into a C-shock; namely

$$
\mathcal{M}_{0}>D_{\max }^{\frac{\gamma_{g}+1}{2}}
$$

The critical Mach number for a C-shock lies in the range 6.35 to 13.39 for $D_{\max }$ between 4 and 7; that is as the net adiabatic index changes from $\gamma_{c}=5 / 3$ to $\gamma_{c}=4 / 3$. It should be noted that this critical sonic Mach number is approximate because $D$ in C-shocks is in general slightly smaller than $D_{\max }$. Therefore, the critical sonic Mach number for C-shock should be slightly smaller than our estimated value. The critical sonic Mach number increases for perpendicular shocks when the magnetic field is strong (Jun et al., 1994). In the 
perpendicular shock case, the condition for a C-shock is $v_{1}^{2}\left(=v_{2}^{2}\right)>c_{1}^{2}\left(=c_{2}^{2}\right)+v_{A, 1}^{2}\left(=v_{A, 2}^{2}\right)$ where $v_{A}$ is the Alfvén speed. This gives the critical sonic Mach number

$$
\mathcal{M}_{0}>\left(D_{\max }^{\gamma_{g}+1}+\frac{v_{A, 0}^{2}}{c_{0}^{2}} D_{\max }^{3}\right)^{1 / 2} .
$$

As long as the gas subshock remains, we can simply relate the states "1" and "2", by the standard shock jump conditions. An assumption in diffusive shock theory is that there is no discontinuity in $P_{c}$, since the CRs necessarily are able to cross the subshock freely. If there is a subshock, we can write

$$
\frac{\rho_{2}}{\rho_{1}}=\frac{\left(\gamma_{g}+1\right) \mathcal{M}_{1}^{2}}{\left(\gamma_{g}-1\right) \mathcal{M}_{1}^{2}+2}=\frac{\left(\gamma_{g}+1\right) \mathcal{M}_{0}^{2}}{\left(\frac{\left(\gamma_{g}-1\right) \mathcal{M}_{0}^{2}}{D^{\gamma_{g}+1}}+2\right) D^{\gamma_{g}+1}} .
$$

If the subshock has vanished $\rho_{2}=\rho_{1}$. Including a subshock the total density jump between states " 0 " and "2" is then

$$
\frac{\rho_{2}}{\rho_{0}}=\frac{\left(\gamma_{g}+1\right) \mathcal{M}_{0}^{2}}{\left(\frac{\left(\gamma_{g}-1\right) \mathcal{M}_{0}^{2}}{D^{\gamma_{g}+1}}+2\right) D^{-\gamma_{g}}} .
$$

If we assume that the Mach number $\mathcal{M}_{0}$ is large compared to the critical Mach number in equation 2 , but there is still a strong subshock equation 5 reduces to

$$
\frac{\rho_{2}}{\rho_{0}} \approx \frac{\gamma_{g}+1}{\gamma_{g}-1} D_{\max }
$$

This last expression cannot apply to a steady shock, but can be approximately true during the evolution of the shock towards its time asymptotic state. For a relatively brief time compared to the evolution time scale of the shock it can even happen that the subshock is strong (so that $\mathcal{M}_{1} \rightarrow \infty$ in equation 4 but that $D \approx D_{\max }$ ). Then the total compression can exceed that of either the precursor or the subshock separately. In fact it can lead to compression as high as 28 (16) for $\gamma_{c}=4 / 3\left(\gamma_{c}=5 / 3\right)$ just as the shock approaches an equilibrium and the spike detaches (the result indicated by equation 6). The extra compression is also accompanied by a modestly enhanced total velocity jump during this time (e.g., Jones \& Kang 1992). This, in turn, can increase the rate of particle acceleration, 
at least for high energy particles whose scattering lengths are large enough to allow them to cross substantial parts of the precursor each time they pass through the subshock (Drury 1987). This influence can speed up the modification process itself as seen in Fig.5 of Jun et al., 1994 .

The overcompression forms a spike, because $\rho_{3}$, if it represents an equilibrium condition for the earlier shock, will satisfy the constraint, $r_{3}=\rho_{3} / \rho_{0} \leq D_{\max }$. Hence, for the reasons we just discussed, there is a time when $\rho_{2}=r_{2} \rho_{0}>\rho_{3}$. We can estimate the width of the spike through a simple consideration of that transition interval. If the time to reestablish equilibrium is $t_{e q}$, then the width of the spike is $x_{s}=\bar{v}_{2} t_{e q}$, where $\bar{v}_{2}$ is the mean velocity relative to the shock inside the density spike. We can assume that the density $\rho_{2}$ peaks in time just before the shock reaches its steady structure and define the total compression at that time to be $r_{2 p}=\rho_{2}($ peak $) / \rho_{0}$. Simplifying the density structure of the spike to a linear profile we can estimate $\bar{v}_{2} \approx \frac{2 v_{0}}{r_{3}+r_{2 p}}$. Then we can use a simple analytic estimate for $t_{e q}$ given by Jones \& Kang (1990) in terms of the upstream diffusion time, $t_{d}=x_{d} / u_{0}=\bar{\kappa} / u_{0}^{2}$, the ratio, $P_{c 2} / P_{c 0}$ of the equilibrium downstream and upstream cosmic-ray pressures and $\gamma_{c}$; namely,

$$
t_{e q} \approx \frac{5 t_{d}}{g} \ln \left[\frac{g\left(P_{c 2} / P_{c 0}\right)+1}{g+1}\right],
$$

where $g=\frac{3}{4} \gamma_{c}-1$. In the limit $g \rightarrow 0, t_{e q} \approx 5 t_{d}\left(P_{c 2} / P_{c 0}-1\right)$. This leads to a simple estimate for the width of the density spike at "separation"; namely,

$$
x_{s} \approx 10 \frac{x_{d}}{g\left(r_{3}+r_{2 p}\right)} \ln \left[\frac{g\left(P_{c 2} / P_{c 0}\right)+1}{g+1}\right],
$$

with an appropriate limit taken as $g \rightarrow 0$. For moderate to strong shocks this formula will generally predict $x_{s} / x_{d} \sim$ few $\times(10-100)$, with larger values for larger $P_{c 2} / P_{c 0}$ and as $\gamma_{c} \rightarrow 4 / 3$ 


\section{One-Dimensional Numerical Simulations}

In order to study the formation process and evolution of the density spike in CR-modified shocks, we use the modified-ZEUS code which solves two-fluid equations on an Eulerian mesh. The algorithms of the code are described in Jun, Clarke, and Norman (1994).

Figure 1 shows the evolution of CR-modified shocks and density spikes computed

for different total Mach numbers defined by $v_{0} / \sqrt{\frac{\gamma_{g} P_{g}}{\rho}+\frac{\gamma_{c} P_{c}}{\rho}}$. To simplify our discussion we have chosen a constant diffusion coefficient, $\kappa=1$ for the moment. The value of the diffusion coefficient enters primarily through its role in determining the width of the density spike (equation 8) and through its role in controlling the rate at which the CR modifications to the shock take place. Figs. 1a, 1b, and 1c show the evolution of CR-modified shocks with Mach number 10, 20, and 50 respectively. The adiabatic index for CRs is $\gamma_{c}=5 / 3$, for these particular tests. Other values will be included later. A shock wave is initially generated by supersonic fluid striking a reflecting boundary at $x=0$ and moves to the right. The dotted lines represent the shock structure at the earliest stage while the later stages are represented by the dashed line and solid lines in a time sequence. The density overshoot is clearly seen in early stages of evolution. This density overshoot results in a "density spike" as the shock evolves further and the gas subshock becomes weaker due to the decreased gas pressure which is replaced by CR pressure. The shock front is strongly modified by the CRs and the gas subshock disappears at later stage of the evolution so that the shock transition becomes continuous (C-shock). The formation of the density spike occurs as the density overshoot lags behind the shock. This lagging starts after temporary high pressure (overshoot) from the shock front generates a positive total pressure gradient and starts to push the density spike downstream. As the overcompressed density spike moves downstream, the shock structure reaches a steady state that can be determined by conservation equations (Drury \& 
Völk 1981, Webb 1983, Jun et al., 1994). The density spike is a moving compression region, and material flows through it. We point out that this feature is not a contact discontinuity. The total compression ratio of the density spike is lower than 16 (for $\gamma_{c}=5 / 3$ ) when it is formed at the shock front. However, after the density spike moves downstream, the compression continues and increases the density further so that it can become higher than 16 for $\gamma_{g}=\gamma_{c}=5 / 3$ (see Fig.1c). The flow compression eventually slows down due to the finite pressure gradient and increasing density. We find in the overcompressed region that the density becomes higher in a stronger shock (higher Mach number). The development of the density spike is not restricted to the piston driven shock problem. Figs. 1d, 1e, and 1f show the evolution of CR-modified shocks which were initialized as a standing shock by assigning analytic values for upstream and downstream states. Total pressure in the downstream is divided equally to gas pressure and CR pressure. The left boundary condition is outflow and the right boundary is inflow with an upstream condition. The results show qualitatively the same density spike evolution as for the CR-modified piston driven shocks. Once detached, the density spike appears to move leftward since the shock front is at rest. The density spike becomes sharper as Mach number increases because the diffusion length is inversely proportional to the shock speed (see equation 8).

In Fig.2, we present the evolution of three different CR-modified shocks. Fig.2a shows the formation of the density spike in a Mach 20 standing shock with the CR adiabatic index $\gamma_{c}=4 / 3$. The shock transition becomes continuous eventually due to the CR domination in the downstream state of the shock. This CR domination of the shock also results in the steady-state density jump of the shock higher than 4 . The prominent density spike is also found in the downstream of the shock as expected. Fig.2b shows the result of CR-modified shock with the diffusion coefficient $\kappa=1 / \rho$. The rest of initial conditions are identical to the case presented in Fig.1d. As reported by Jun et al. (1994), the steady state is obtained faster than in the case with $\kappa=1$. Another feature is the sharper structure of the shock 
and density spike because of the smaller diffusion length in the shock transition region and downstream state where the density is higher than 1. Fig.2c shows the evolution of CR-modified shock which contains a gas subshock and a smooth precursor. The density spike is also clearly present, although it is not as prominent as in C-shock case. This example manifests that the density spike is not restricted to the C-shock case. This density enhancement always follows the formation or enhancement of a precursor. Therefore, the density augmentation should form whenever the CR acceleration is efficient and the back-reaction of CR to the shock front is dynamically important.

The density spike also forms in simulations involving shock propagation into nonuniform media. It has been seen in cases where the shock moves down a steep density gradient, such as a SNR blast into a preexisting wind cavity, or where a CR-modified shock encounters a steep increase in upstream density, such as a cloud. Fig.3 shows the evolution of a Mach 10 shock propagating into a higher density background. The shock is initialized as a standing shock at $\mathrm{x}=3$. The shock is already modified by generating a precursor and density overshoot at $\mathrm{t}=0.1$. By $\mathrm{t}=0.2$, a density spike has formed and is moving to the left relative to the shock front. The shock transition has reached a steady state and the gas subshock disappeared (leaving a C-shock). At $\mathrm{t}=0.3$, the C-shock is approaching a higher density medium $(\rho=3)$, while at $\mathrm{t}=0.4$, the $\mathrm{C}$-shock is interacting with the higher density region and another density overshoot is produced. Finally, at $\mathrm{t}=0.5$, the shock reached to the new steady state and the second density spike is formed. The C-shock structure is recovered (Figs. 3e and 3f).

As the CR-modified shock encounters the denser external medium, its precursor is compressed and the shock structure temporarily reforms a viscous, gas subshock. This process can occur if the rate of increase of the incoming gas density exceeds the rate at which the shock is able to adjust to an equilibrium in terms of its CR pressure, as 
given by equation 7 . As the newly formed discontinuous shock propagates into the denser background, the shock is modified anew and the precursor is enhanced. The important effect during the shock passage into the denser region is a higher acceleration efficiency due to increased compression. If the inhanced upstream density is in pressure equilibrium with its surroundings, it will be colder. Then it is easy to show that the Mach number of the shock increases as it penetrates the denser medium. The importance of this will be more significant in an already CR-dominated C-shock, because the full shock compression can become much higher in responce to the density enhancement encounter than through the original incoming C-shock. Jones and Kang (1992) found in their shock-cloud two-fluid simulations that the acceleration efficiency is increased more by the encounter if the incident shock is previously dominated by CR pressure.

In the begining of the density spike formation, the total pressure decreases upstream of the density spike in order to conserve total momentum flux $\left(\rho v^{2}+P_{g}+P_{c}\right)$. As a result, the total pressure in the left side of the density spike has a negative gradient while the density gradient is positive. As we will show in the next section, these opposite gradients between the total pressure and the density consitute an unstable condition. As the shock structure itself reaches a dynamical steady state (equilibrium), the momentum flux upstream of the density spike becomes constant and the steepening of the density spike slows down. Eventually, the gradients of the total pressure and the density decreases, after the density spike is detached from the shock front, mainly because of CR diffusion. Once this happens, the growth rate of the instability decreases accordingly. From this dynamical picture it is obvious that the duration of the instability is comparable to the shock equilibrium timescale (equation 7) each time the shock readjusts. Since the opposite gradients of the total pressure and the density exist only for a short period of time during the formation of the density spike, the driving force of the instability appears to be impulsive. 


\section{Modified Rayleigh-Taylor Instability}

One can expect that cosmic-ray pressure should be added to the total pressure in the criterion for the Rayleigh-Taylor instability in the presence of cosmic-ray particles. In this section, we derive the modified criterion for the Rayleigh-Taylor instability in the presence of CR pressure by considering the two-fluid model (Drury \& Völk 1981). The governing equations are:

$$
\begin{gathered}
\frac{\partial \rho}{\partial t}+\nabla \cdot(\rho \vec{v})=0 \\
\rho \frac{\partial \vec{v}}{\partial t}+\rho(\vec{v} \cdot \nabla) \vec{v}+\nabla\left(P_{g}+P_{c}\right)+\rho \vec{g}=0 \\
\frac{\partial P_{g}}{\partial t}+\vec{v} \cdot \nabla P_{g}+\gamma_{g} P_{g} \nabla \cdot \vec{v}=0 \\
\frac{\partial P_{c}}{\partial t}+\vec{v} \cdot \nabla P_{c}+\gamma_{c} P_{c} \nabla \cdot \vec{v}=\nabla \cdot\left(\kappa \nabla P_{c}\right),
\end{gathered}
$$

where $P_{c}$ is the $\mathrm{CR}$ pressure and $\kappa$ is the mean spatial $\mathrm{CR}$ diffusion coefficient. The gravity term is added to the momentum equation to satisfy the equilibrium state in a stationary condition. For this analytical analysis we assume $\kappa$ to be a constant, but that is not necessary, either for this instability, or for validity of the two-fluid model (see Kang et al., 1992; Kang \& Jones 1995; Kang \& Jones 1996 for specific tests of time-dependent two-fluid simulations against more detailed diffusion-convection equation or "particle" simulations).

We consider an exponential background,

$$
\bar{v}_{x}=v_{0} \exp \left(\frac{x-x_{0}}{L_{1}}\right), \quad \bar{\rho}=\rho_{0} \exp \left(\frac{x-x_{0}}{L_{2}}\right), \quad \bar{P}_{g}=P_{g 0} \exp \left(\frac{x-x_{0}}{L_{3}}\right), \quad \bar{P}_{c}=P_{c 0} \exp \left(\frac{x-x_{0}}{L_{4}}\right) .
$$

In general, the normal mode for a nonuniform background contains a dependence on the $\mathrm{x}$-direction, and one needs to solve the characteristic value problem with proper boundary conditions. However, since we are seeking the instability criterion only, we can restrict our analysis to the local region, $x-x_{0} \ll L_{1}$. We consider plane wave perturbations, $\sim \psi_{1} \exp \left(n t+i k_{y} y\right)$ where $\psi_{1}$ is a perturbed quantity $\left(v_{x 1}, v_{y 1}, \rho_{1}, P_{g 1}\right.$, or $\left.P_{c 1}\right)$. Then, one 
can obtain the linearized perturbation equations using equations 9 - 13,

$$
\begin{gathered}
\rho_{1}\left(n+\frac{v_{0}}{L_{1}}\right)+i k_{y} \rho_{0} v_{y 1}+\frac{\rho_{0}}{L_{2}} v_{x 1}=0, \\
v_{x 1}\left(n+\frac{v_{0}}{L_{1}}\right)-\frac{\rho_{1}}{\rho_{0}^{2}}\left(\frac{P_{g 0}}{L_{3}}+\frac{P_{c 0}}{L_{4}}\right)=0, \\
n v_{y 1}+\frac{i k_{y}}{\rho_{0}}\left(P_{g 1}+P_{c 1}\right)=0, \\
P_{g 1}\left(n+\gamma_{g} \frac{v_{0}}{L_{1}}\right)+v_{x 1} \frac{P_{g 0}}{L_{3}}+i k_{y} \gamma_{g} P_{g 0} v_{y 1}=0, \\
P_{c 1}\left(n+\gamma_{c} \frac{v_{0}}{L_{1}}+\kappa k_{y}^{2}\right)+v_{x 1} \frac{P_{c 0}}{L_{4}}+i k_{y} \gamma_{c} P_{c 0} v_{y 1}=0 .
\end{gathered}
$$

By combining the above equations, we get the dispersion relation,

$$
\begin{aligned}
& \left(\rho_{0}\left(n+\frac{v_{0}}{L_{1}}\right)^{2}+\frac{1}{L_{2}}\left(\frac{P_{g 0}}{L_{3}}+\frac{P_{c 0}}{L_{4}}\right)\right) \\
\times & \left(n\left(n+\frac{\gamma_{g} v_{0}}{L_{1}}\right)\left(n+\frac{\gamma_{c} v_{0}}{L_{1}}+\kappa k_{y}^{2}\right)+k_{y}^{2} a_{g}^{2}\left(n+\frac{\gamma_{c} v_{0}}{L_{1}}+\kappa k_{y}^{2}\right)+k_{y}^{2} a_{c}^{2}\left(n+\frac{\gamma_{g} v_{0}}{L_{1}}\right)\right) \\
- & \frac{k_{y}^{2} P_{g 0}}{\rho_{0} L_{3}}\left(n+\frac{\gamma_{c} v_{0}}{L_{1}}+\kappa k_{y}^{2}\right)\left(\frac{P_{g 0}}{L_{3}}+\frac{P_{c 0}}{L_{4}}\right)-\frac{k_{y}^{2} P_{c 0}}{\rho_{0} L_{4}}\left(n+\frac{\gamma_{g} v_{0}}{L_{1}}\right)\left(\frac{P_{g 0}}{L_{3}}+\frac{P_{c 0}}{L_{4}}\right)=0
\end{aligned}
$$

where $a_{g}=\left(\frac{\gamma_{g} P_{g 0}}{\rho_{0}}\right)^{1 / 2}$, and $a_{c}=\left(\frac{\gamma_{c} P_{c 0}}{\rho_{0}}\right)^{1 / 2}$.

Let us consider two different limits of this dispersion relation in a stationary background $\left(v_{0} \rightarrow 0\right)$.

\subsection{Short-Wavelength Limit $\left(k_{y} \rightarrow \infty\right)$}

The familiar and related Rayleigh-Taylor and convective instabilities in pure gas dynamics are actually somewhat different in terms of criteria, because the convective instability depends on fluid compressibility, whereas the classical Rayleigh-Taylor instability does not. A flow is convectively unstable if $\frac{\partial \ln \bar{\rho}}{\partial x} / \frac{\partial \ln \bar{P}_{g}}{\partial x}<1 / \gamma_{g}$, while the flow is RayleighTaylor unstable if $\frac{\partial \ln \bar{\rho}}{\partial x} / \frac{\partial \ln \bar{P}_{g}}{\partial x}<0$ (Bandiera 1984). This shows that the convective instability is present in a wider range of conditions than the Rayleigh-Taylor instability. When the CR 
diffusion term is ignored, our dispersion relation reduces to the growth rate for the general convective instability in the short-wavelength limit (for the frequency range, $n \gg \kappa k_{y}^{2}$ but $\left.n^{2} \ll k_{y}^{2}\left(a_{g}^{2}+a_{c}^{2}\right)\right)$.

$$
n^{2}=\frac{1}{\rho_{0}^{2}\left(a_{g}^{2}+a_{c}^{2}\right)}\left(\frac{P_{g 0}}{L_{3}}+\frac{P_{c 0}}{L_{4}}\right)^{2}-\frac{1}{\rho_{0} L_{2}}\left(\frac{P_{g 0}}{L_{3}}+\frac{P_{c 0}}{L_{4}}\right) \simeq \frac{1}{\gamma_{t} \rho_{0}}\left(\frac{\partial \bar{P}_{t}}{\partial x}\right)\left(\frac{\partial \ln \left(\bar{P}_{t} / \bar{\rho}^{\gamma_{t}}\right)}{\partial x}\right) .
$$

where $\gamma_{t}=\frac{\gamma_{g} P_{g 0}+\gamma_{c} P_{c 0}}{P_{g 0}+P_{c 0}}$ and $\bar{P}_{t}=\bar{P}_{g}+\bar{P}_{c}$.

In the presence of CR diffusion and short wavelength limit (for the frequency range, $n \ll \kappa k_{y}^{2}$ and $n^{2} \ll k_{y}^{2}\left(a_{g}^{2}+a_{c}^{2}\right)$, the dispersion relation reduces to

$$
n^{2}=\frac{1}{\rho_{0}}\left(\frac{P_{g 0}}{L_{3}}+\frac{P_{c 0}}{L_{4}}\right)\left(\frac{1}{\gamma_{g} L_{3}}-\frac{1}{L_{2}}\right) \simeq \frac{1}{\rho_{0}} \frac{\partial \bar{P}_{t}}{\partial x}\left(\frac{1}{\gamma_{g}} \frac{\partial \ln \bar{P}_{g}}{\partial x}-\frac{\partial \ln \bar{\rho}}{\partial x}\right) .
$$

This relation includes criteria for both gas convection and Rayleigh-Taylor instabilties. Now, let us consider our dispersion relation for several different cases.

i) First, when all three gradients $\left(\partial \bar{P}_{t} / \partial x, \partial \bar{P}_{g} / \partial x\right.$, and $\left.\partial \bar{\rho} / \partial x\right)$ have same sign, then the flow can be convectively unstable if $\left|\frac{1}{\gamma_{g}} \frac{\partial \ln \overline{P_{g}}}{\partial x}\right|>\left|\frac{\partial \ln \bar{\rho}}{\partial x}\right|$.

ii) Second, the flow is always Rayleigh-Taylor unstable if

$$
\frac{\partial \bar{P}_{t}}{\partial x}>0, \frac{\partial \bar{P}_{g}}{\partial x}>0, \frac{\partial \bar{\rho}}{\partial x}<0, \quad \text { or } \quad \frac{\partial \bar{P}_{t}}{\partial x}<0, \frac{\partial \bar{P}_{g}}{\partial x}<0, \frac{\partial \bar{\rho}}{\partial x}>0 .
$$

iii) Third, the flow is Rayleigh-Taylor unstable if

$$
\frac{\partial \bar{P}_{t}}{\partial x}>0, \frac{\partial \bar{P}_{g}}{\partial x}<0, \frac{\partial \bar{\rho}}{\partial x}<0, \quad \text { or } \quad \frac{\partial \bar{P}_{t}}{\partial x}<0, \frac{\partial \bar{P}_{g}}{\partial x}>0, \frac{\partial \bar{\rho}}{\partial x}>0
$$

when the gas is convectively stable $\left(\left|\frac{1}{\gamma_{g}} \frac{\partial \ln \bar{P}_{g}}{\partial x}\right|<\left|\frac{\partial \ln \bar{\rho}}{\partial x}\right|\right)$. It is interesting to see that the convective instability can be suppressed by the opposite CR pressure gradient. 


\subsection{Large-Wavelength Limit $\left(k_{y} \rightarrow 0\right)$}

In the large-wavelength limit (for the range, $n \gg \kappa k_{y}^{2}$ and $n^{2} \gg k_{y}^{2}\left(a_{g}^{2}+a_{c}^{2}\right)$ ), the dispersion relation reduces to

$$
n^{2}=-\frac{1}{L_{2} \rho_{0}}\left(\frac{P_{g 0}}{L_{3}}+\frac{P_{c 0}}{L_{4}}\right) \simeq-\frac{1}{\rho_{0}^{2}} \frac{\partial \bar{\rho}}{\partial x} \frac{\partial\left(\bar{P}_{g}+\bar{P}_{c}\right)}{\partial x} .
$$

Therefore, the flow is unstable if

$$
\frac{\partial \bar{\rho}}{\partial x} \frac{\partial \bar{P}_{t}}{\partial x}<0
$$

This is the generalized crossed-gradient condition for Rayleigh-Taylor instability when the total pressure is considered instead of the gas pressure (e.g. see Jones et al., 1981). One can see that the convective instability disappears in the large-wavelength limit.

From the above analysis, one can see that the flow is Rayleigh-Taylor unstable throughout the entire wavelength range when the total pressure and gas pressure gradients are in opposite sign to the density gradient, which is the instability condition in our density spike case.

\section{Two-Dimensional Numerical Results and Discussion}

The nonlinear development of the unstable density spike is best studied by means of multi-dimensional numerical simulations. We have simulated the instability of the density spike in two dimensions by setting up several different CR-modified shocks, such as piston-driven shocks and standing shocks. The density spike is found to be unstable in every case. To reduce the potential that we are observing only a numerical effect, we carried out analogous simulations with two very different codes; namely a version of ZEUS, modified to include two-fluid CR physics (Jun et al., 1994) and a version of PPM, also modified and used extensively in the past for a number of CR applications (e.g., Jones 
\& Kang 1990; Jones \& Kang 1993; Ryu, Kang \& Jones 1993). The gasdynamical methods are quite distinct in the two codes, and the CR transport methods are also different (one being explicit and one being implicit, for example). The results of the two codes were qualitatively the same, and most importantly, both demonstrated the instability. Fig. 4 shows the two-dimensional results of a Mach 10 CR-modified shock structures computed by the modified ZEUS code. The one-dimensional time-dependent evolution of the same shock is shown in Fig.1d. The computational space is resolved by 600x200 uniform zones. A random density perturbation $(10 \%)$ is carried in by the flow from the right boundary. The density perturbation (pressure-free perturbation) is chosen because we do not intend to generate sound waves that are unstable in the CR-dominated shock (acoustic instability, see, e.g., Drury \& Falle 1986; Kang et al., 1992). In this way, we should be free of any influence from the propagating acoustic instability, so that the instability we observe in the density spike results only from the Rayleigh-Taylor instability.

Images in Fig. 4 show the density at $\mathrm{t}=0.1,0.2$, and 0.3 (top to bottom). A number of thin fingers are produced in the left side of the density spike as a result of the instability. In addition, these fingers show unstable curly structures that are likely to be the result of the secondary Kelvin-Helmholtz instability which was triggered by the shear between the finger and the background flow downstream of the Rayleigh-Taylor unstable region. The evolution of the unstable flow is found to be different from the classical Rayleigh-Taylor instability in the following way. In the classical Rayleigh-Taylor instability, short wavelength modes appear first in the linear regime, because their growth rates are higher. However, the nonlinear Rayleigh-Taylor instability is generally dominated by larger wavelength features, because they reach higher terminal velocity and overtake smaller structures (Jun et al., 1995). However, short wavelengths are still dominant in the nonlinear stage of our CR-modified shock case. This is because the unstable condition exists only temporarily and does not provide sufficient time for growth of perturbations on the scale of the thickness 
of the density spike itself. (Recall that the perturbation wavevectors are transverse to the flow direction.) Short wavelength features are just stretched along the flow direction by the initial acceleration to form long thin fingers without much interaction with one another.

To provide a sense of the time evolution of the instability, Fig.5 shows the amplitude of the density fluctuations as a function of time. The amplitude, $\mathrm{A}(\mathrm{x})$ is defined as $\frac{1}{H} \int_{O}^{H} \delta \rho(x, y)^{2} d y$ where $H$ is the width of the computational plane in the Y-direction and $\delta \rho(x, y)=\frac{\rho(x, y)}{\rho(x)}-1$. This amplitude is averaged over the region $x_{2}-x_{1}$ in the unstable flow; that is, $A=\frac{1}{x_{2}-x_{1}} \int_{x_{1}}^{x_{2}} A(x) d x$. The domain for the average is chosen to be 10 zones leftward from the peak density in the density spike. The amplitude, A provides us some information about the overall density fluctuation history. We find that Fourier amplitude of the density perturbation is not a good way to measure the growth of the instability because the flow is moving through the unstable region.

The apparent behavior of $\mathrm{A}(\mathrm{x})$ allows us to divide the growth history of the instability into 4 different stages as indicated in Fig.5. In stage 1, the flow carrying density perturbation has not reached the density spike. The amplitude of the density perturbation increases suddenly in stage 2 due to the incoming density perturbation. The density perturbation grows while the flow with the density perturbation passes through the unstable region (stage 3 ). Recall that the growth of the instability exists only temporarily while the flow in the left (downstream) side of the density spike is unstable. The, final, relaxation phase of the instability is shown in stage 4 . The sudden decay of the instability growth is likely due to the combined effects of relaxation of the unstable region and the nonlinearity of the instability. The instability is found to result in a slight reduction in density in the spike compared to the one-dimensional behavior at this time. However, this reduction is small because of the short duration of unstable flow at the early stage.

Although our perturbations were designed to avoid generation of upstream sound 
waves that might contribute to this instability, we recognized that passage of the density perturbations through the shock might also produce sonic perturbations. To avoid any possibility that this might modify the instability of interest, we have done a simulation with the diffusion coefficient, $\kappa=1 / \rho$, because it is known that CR-modified shocks are stable against the accoustic instability when $\kappa$ takes this form (Drury \& Falle 1986). Fig.6 shows a greyscale image of a two-dimensional numerical result for the case with $\kappa=1 / \rho$ at $\mathrm{t}=0.2$ (one-dimensional result for this case is presented in Fig.2b). The density spike is still unstable, thus, confirming the existence of the physical Rayleigh-Taylor instability in the density spike. The shorter density fingers compared to Fig.4b are due to the narrower density spike caused by the reduction in diffusion length and equilibrium time when $\kappa=1 / \rho$ (compare Fig.1d and Fig.2b). In the presence of sonic perturbations, Ryu, Kang \& Jones 1993 discovered in two-dimensions there is a secondary, Rayleigh-Taylor class instability associated with the nonlinear accoustic instability, which generates accoustic turbulence within the shock precursor. Since that instability will also act as strong CR-modified shocks come to equilibrium, it may be natural to expect that the density spike will often be subject to strong perturbations.

An obvious place where one can expect the unstable density spike to be formed may be in the supernova remnant blast wave structure. Numerical studies of CR-modified supernova remnant shocks reveal the appearance of the density spike in the evolution (Dones \& Kang 1992). Stronger modification of the shock structure by CR pressure was found in the simulations when the supernova remnant shock propagates into an inverse square density medium such as a wind-blown background. Therefore, this density spike could be found in the early stage of supernova remnant evolution while the supernova shock is still inside the presupernova wind. Discovery of this unstable density spike and consequent turbulent flow would provide strong, in situ evidence within supernova remnants for the action of diffusive shock acceleration and, especially for strong shock modification by 
energetic particles. We note finally that, although these effects are strongest for C-shocks, the development of C-shocks is not necessary for what we have discussed in this paper. It is necessary, however, that CR pressure become the dominant means of decelerating the flow through the shock.

\section{Summary}

We have given a detailed description and numerical study of the density spike formation downstream of a CR-modified shock structure. The density spike originates from a temporary overcompression while the gas shock is modified by the CR pressure. The resulting compression ratio is found to increase with the flow Mach number. We emphasize that the density spike is a common relic of the shock modification by the CR pressure. The density spike is found to be Rayleigh-Taylor unstable at the early stages of its evolution. Our linear analysis shows that the flow is Rayleigh-Taylor unstable if the total pressure gradient and gas density gradient are of opposite signs. We have simulated this unstable density spike by solving the time dependent two-fluid equations numerically on a two-dimensional grid. It is found that the instability grows impulsively in the early stages, but slows down as the density spike begins to relax its structrure. The numerical results show a number of thin fingers growing downstream from the density spike. However, the mass density in the spike is decreased only slightly as a result of the weak instability.

The simulations were performed on a Cray C90 at the Minnesota Supercomputer Center. The work reported here is supported in part by NSF grant AST-9318959 by NASA grants NAGW-2548 and NAG5-5055 and by the Minnesota Supercomputer Institute. 


\section{REFERENCES}

Axford, W.I., Leer, E., and Skadron, G. 1977, Proc. 15th Internat. Cosmic Ray Conf(Plovdiv), 11, 132

Bandiera, R. 1984, A\&A, 139, 368

Bell, A. R. 1978, MNRAS, 182, 147

Blandford, R. D., \& Ostriker, J. P. 1978, ApJ, 221, L29

Blandford, R. D. 1980, ApJ, 238, 410

Dorfi, E. A. 1984, Adv. Space Res., 4, 205

Dorfi, E. A. 1985, in Cosmical Gas Dynamics, ed. F. D. Kahn (Utrecht: VNU Science Press), p. 137

Drury, L.O'C. 1987, Proc. Sixth Int. Solar Wind Conf., ed. U. J. Pizza, T. Holzer, and D. G. Sime (NCAR/TN-360+proc), p.521

Drury, L.O'C. \& Falle, S. A. E. G. 1986, MNRAS, 223, 353

Drury, L. O’C., Markiewicz, W. J. \& Völk, H. J. 1989, A\&A, 225, 179

Drury, L.O'C. \& Völk, H. J. 1981, ApJ, 248, 344

Ellison, D. C., Möbius, E. \& Paschmann, G. 1990, ApJ, 352, 376

Frank, A., Jones, T. W. \& Ryu, D. 1995, ApJ, 441, 629

Jones, F. C. \& Ellison, D. C. 1991, Space Sci. Rev., 58, 259

Jones, T. W. \& Kang, H. 1990, ApJ, 363, 499

Jones, T. W. \& Kang, H. 1992, ApJ, 396, 575 
Jones, T. W. \& Kang, H. 1993, ApJ, 402, 560

Jones, E. M., Smith, B. W., \& Straka, W. C. 1981, ApJ, 249, 185

Jun, B.-I., Clarke, D. A., \& Norman, M. L. 1994, ApJ, 429, 748

Jun, B.-I., Norman, M. L., \& Stone, J. M. 1995, ApJ, 453, 332

Kang, H. \& Jones, T. W. 1990, ApJ, 353, 149

Kang, H., Jones, T. W., \& Ryu, D. 1992, ApJ, 385, 193

Kang, H. \& Jones, T. W. 1995, ApJ, 447, 944

Kang, H. \& Jones, T. W. 1996, ApJ, (in press)

Krymsky, G. F. 1977, Dok. Acad. Nauk. USSR, 234, 1306

Malkov, M. A. \& Völk H. J. 1996, ApJ, (in press)

Ryu, D., Kang, H. \& Jones, T. W. 1993, ApJ, 405, 199

Webb, G. M. 1983, A\&A, 127, 97

Webb, G. M., Drury, L. O'C., \& Völk, H. J. 1986, A\&A, 160, 335 
Fig. 1.- Density evolution of CR-modified shock structures for various Mach numbers. The shock is propagating into a uniform background of $\rho=1, P_{g}=1, P_{c}=1$. Adiabatic index, $\gamma$, is assumed to be $5 / 3$ for both gas and CR and the diffusion coefficient, $\kappa=1$. Each plots (dotted line, dashed line, and solid line) are represented in a time sequence. The computational domain is resolved by 300 uniform grid zones. Readers are referred to Dorfi(1984,1985), Jones \& Kang(1990), and Jun et al. (1994) for the evolution of other dynamical variables. (a) Piston driven shock with Mach 10. The shock is propagating with the velocity $v_{s}=18.257$. Three density plots are taken at $\mathrm{t}=0.1,0.2$, and 0.3 . (b) Piston driven shock with Mach $20\left(v_{s}=36.515\right)$. Three density plots are taken at $\mathrm{t}=$ 0.033, 0.066, and 0.099. (c) Piston driven shock with Mach 50 ( $\left.v_{s}=91.287\right)$. Three density plots are taken at $\mathrm{t}=0.008,0.016$, and 0.024. (d) Standing shock with Mach 10 $\left(v_{s}=18.257\right)$. The upstream $\left(\rho=1.0, v=-18.257, P_{g}=1.0, P_{c}=1.0\right)$ and downstream $\left(\rho=3.884, v=-4.7, P_{g}=124.75, P_{c}=124.75\right)$ states of the flow are initialized with analytic jump condition of CR-modified shock. Total pressure in the downstream is divided equally to gas pressure and CR pressure. Three density plots are taken at $t=0.1,0.2$, and 0.3 . (e) Standing shock with Mach 20 (upstream state: $\rho=1.0, v=-36.515, P_{g}=1.0, P_{c}=1.0$. downstream state: $\left.\rho=3.97, v=-9.197, P_{g}=499.75, P_{c}=499.75\right)$. Three density plots are taken at $\mathrm{t}=0.033,0.066$, and 0.099. (f) Standing shock with Mach 50 (upstream state: $\rho=1.0, v_{s}=-91.287, P_{g}=1.0, P_{c}=1.0$. downstream state: $\rho=3.995, v_{s}=-22.847, P_{g}=$ $3124.7, P_{c}=3124.7$ ). Three density plots are taken at $\mathrm{t}=0.008,0.016$, and 0.024 .

Fig. 2.- (a) Standing CR-modified shock (Mach 20, $v_{s}=34.641$ ). The adiabatic index for $\mathrm{CR} \gamma_{c}=4 / 3$ and $\kappa=1$. Plots are taken at $\mathrm{t}=0.1$ (dotted line), $\mathrm{t}=0.2($ dashed line), and $\mathrm{t}=0.3$ (solid line). The numerical resolution of the entire computational space is 300 zones. (b) Standing CR-modified shock (Mach 10) with the diffusion coefficient $\kappa=1 / \rho$. Other physical conditions are identical to the case in Fig.1d. (c) Standing CR-modified shock (Mach 5, $v_{s}=8.66, \gamma_{c}=4 / 3$ ). The computational space is resolved by 800 grid zones. 
Fig. 3.- Mach 10 shock $\left(v_{s}=18.257\right)$ is propagating into the higher density region $(\rho=3)$. The adiabatic index for $\mathrm{CR}$ is $\gamma_{c}=5 / 3$, and $\kappa=1$. The computational space is resolved by 400 grid zones.

Fig. 4.- Grey-scale images show the density of two-dimensional numerical result of CRmodified standing shock with Mach 10 at $\mathrm{t}=0.1,0.2$, and 0.3 (from top to bottom). The computational space is resolved by 600x200 uniform grid zones. One-dimensional result is shown in Fig.1d.

Fig. 5.- The growth of the density perturbation in the density spike in Mach 10 standing shock (fig.4).

$$
A(x)=\frac{1}{H} \int_{0}^{H} \delta \rho(x, y)^{2} d y
$$

where $H$ is the width of the computational plane in Y-direction, $\delta \rho(x, y)=\frac{\rho(x, y)}{\rho(x)}-1$, and $\rho \overline{(x)}$ is the averaged density over Y-direction.

$$
A=\frac{1}{x_{2}-x_{1}} \int_{x_{1}}^{x_{2}} A(x) d x
$$

where the average is taken over the region $x_{2}-x_{1}$ in the unstable flow.

Fig. 6.- Grey scale image of CR-modified shock generated by Mach 10 shock at t=0.2. The diffusion coefficient used is $1 / \rho$. Other physical parameters are identical to the ones used Fig.2b case. The computational space is resolved by 600x200 uniform grid zones. 
(a)

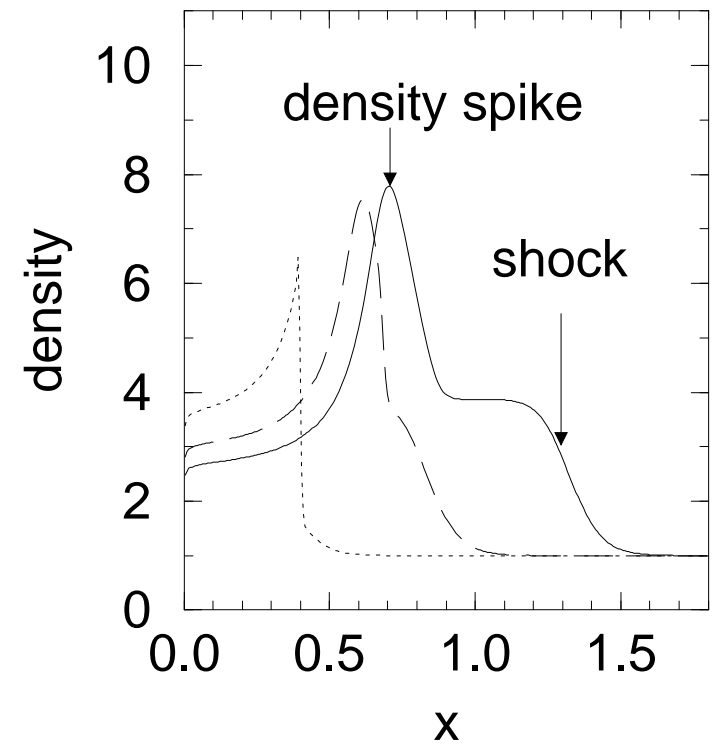

(d)

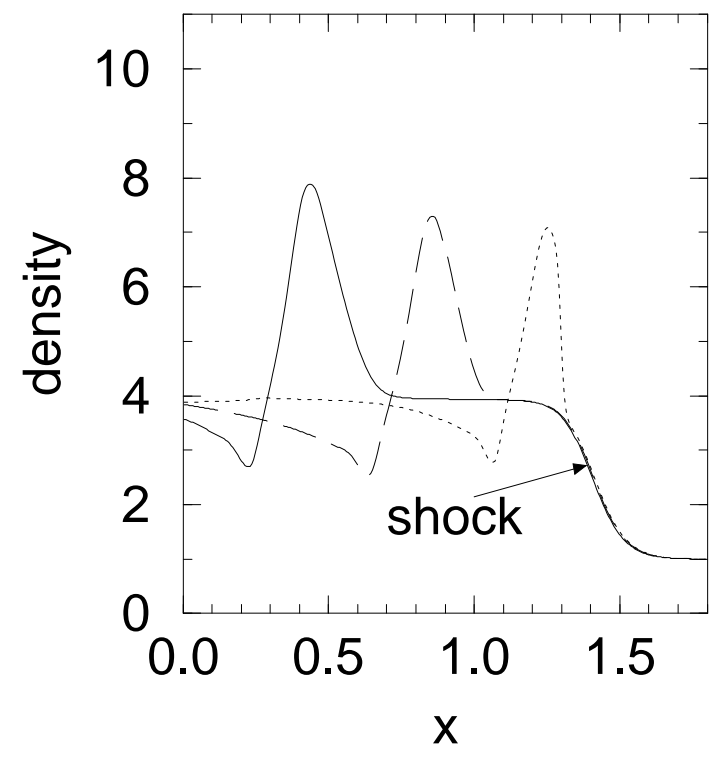

(b)

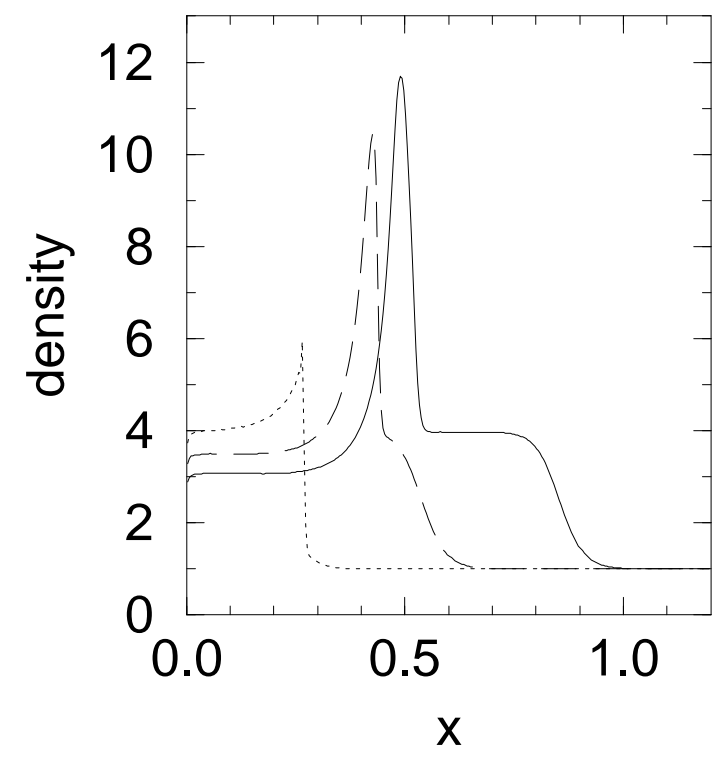

(e)

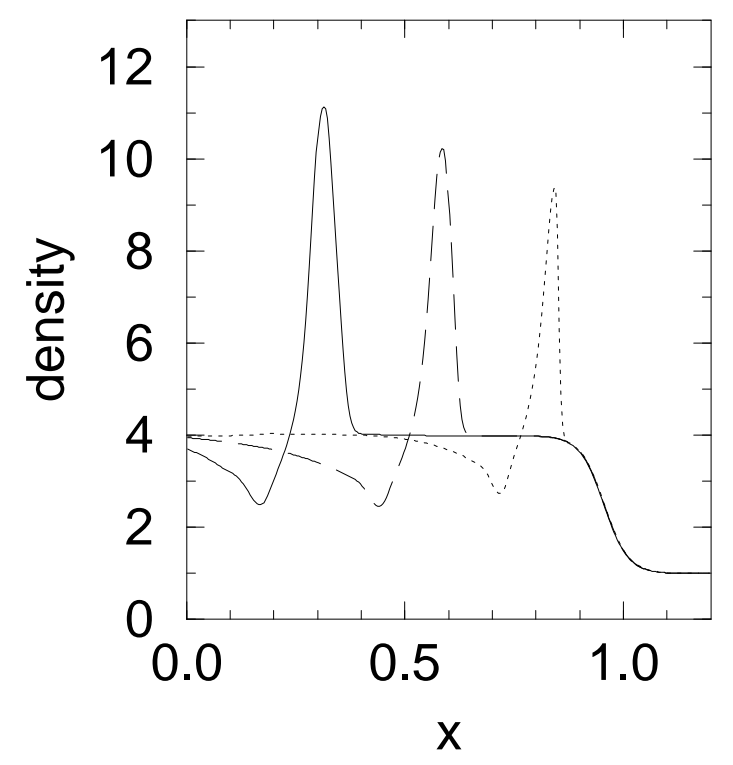

(c)

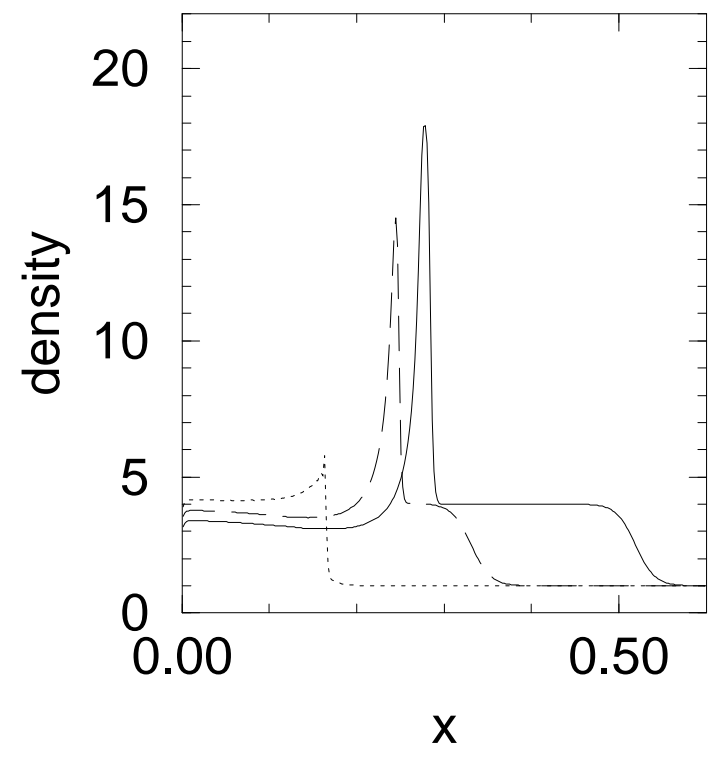

(f)

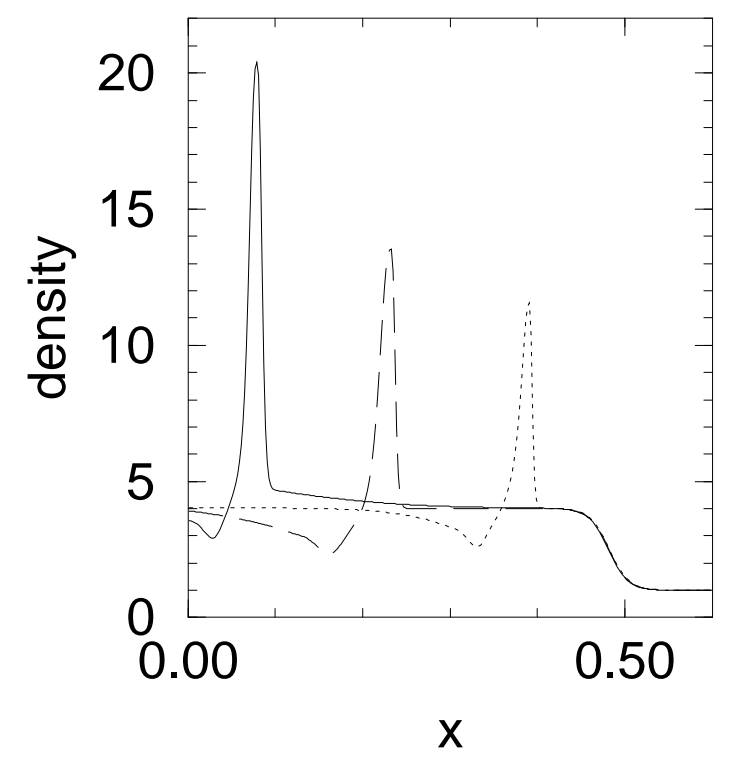


(a)

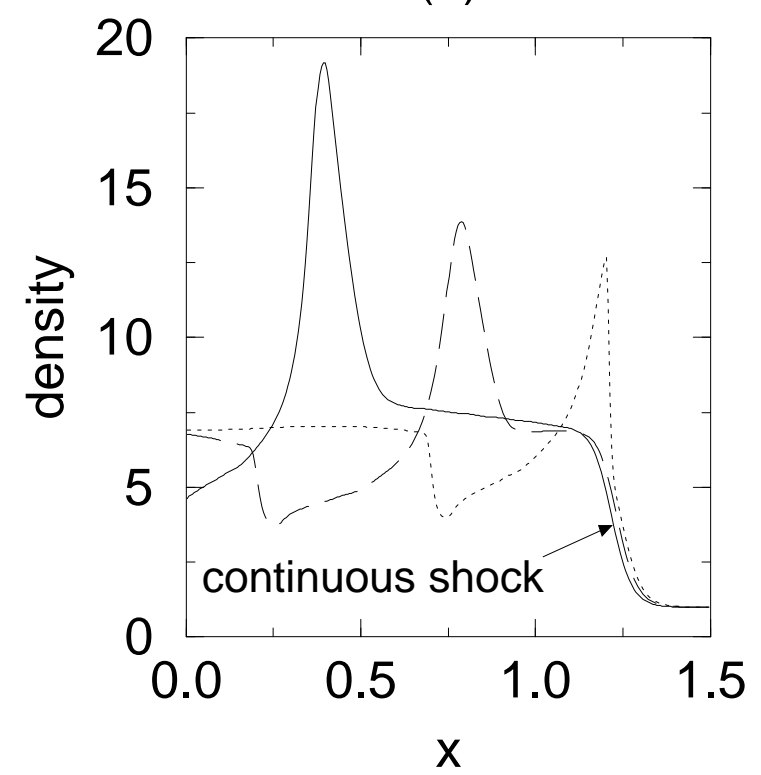

(b)

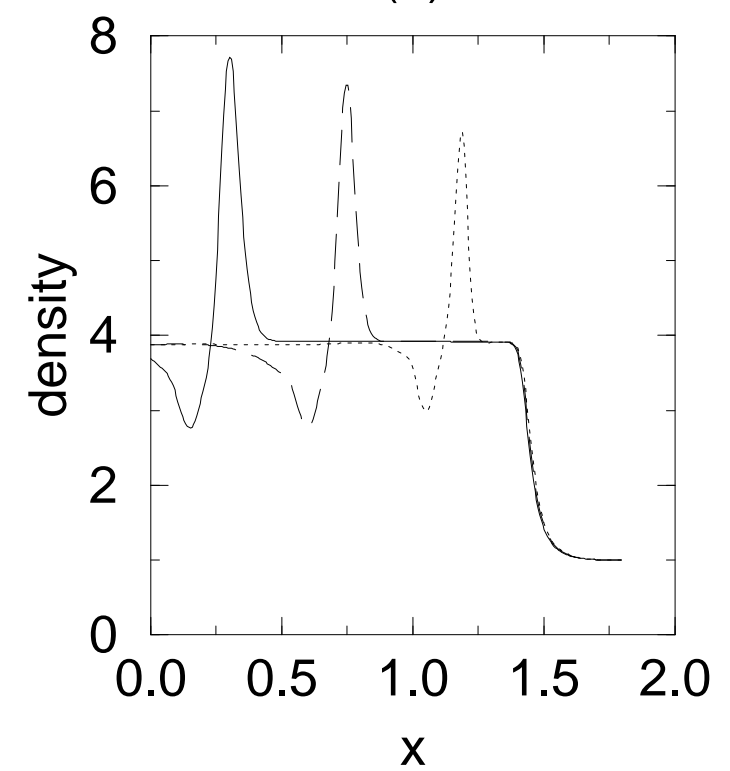

(c)

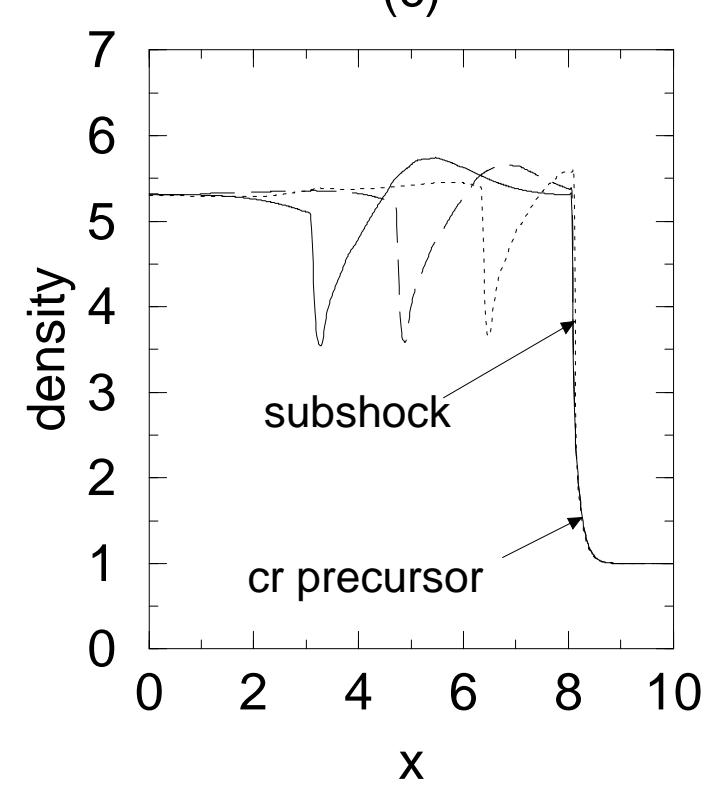


(a) $t=0.1$

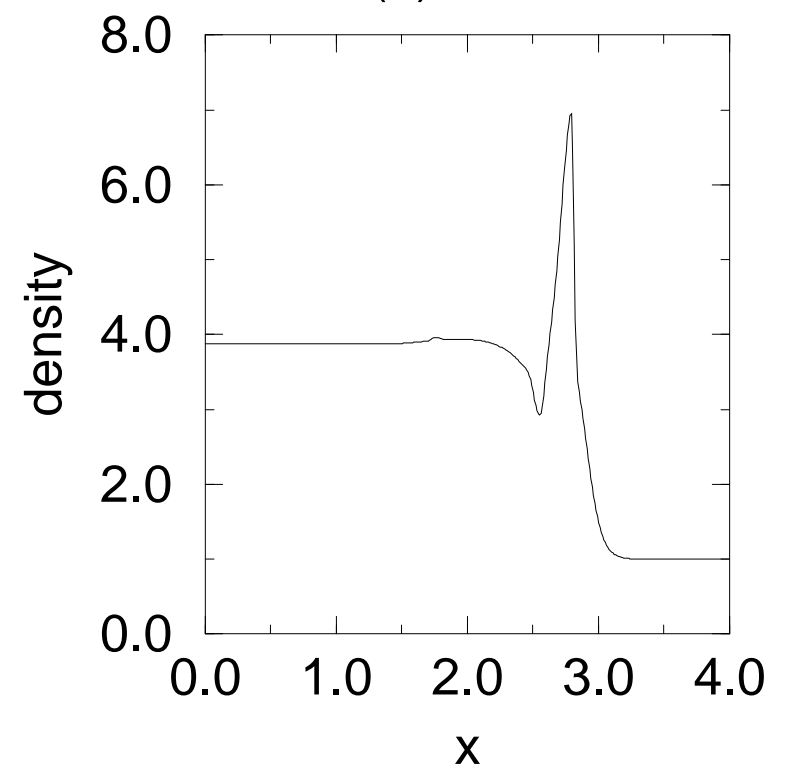

(d) $t=0.4$

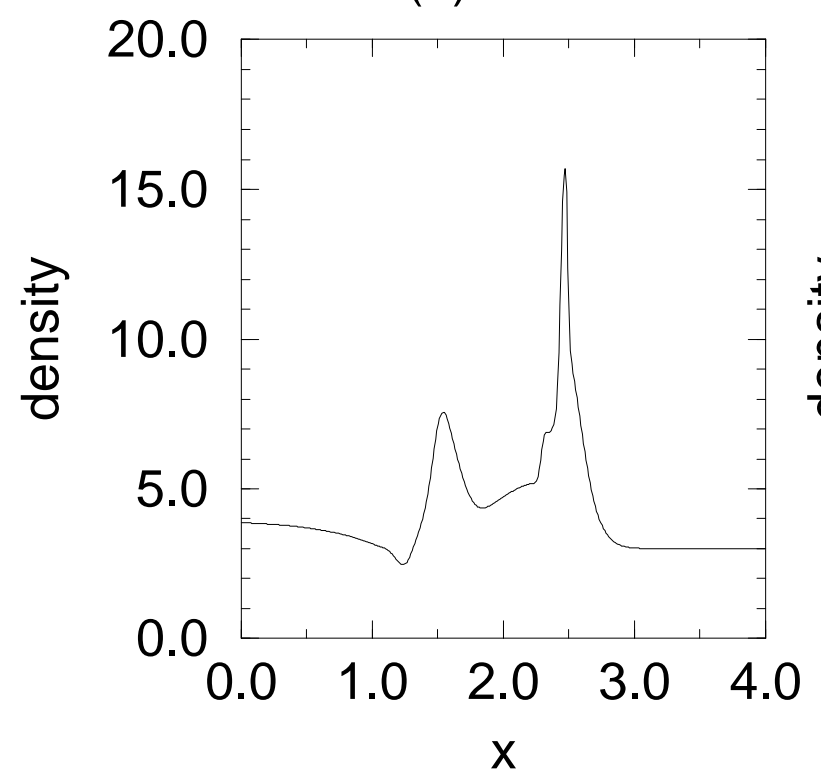

(b) $t=0.2$

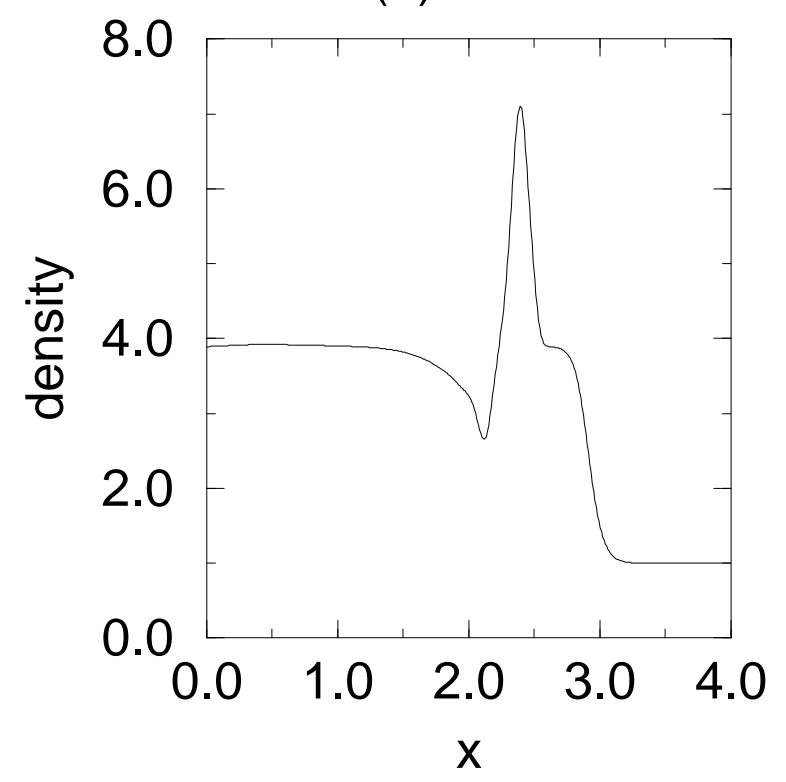

(e) $t=0.5$

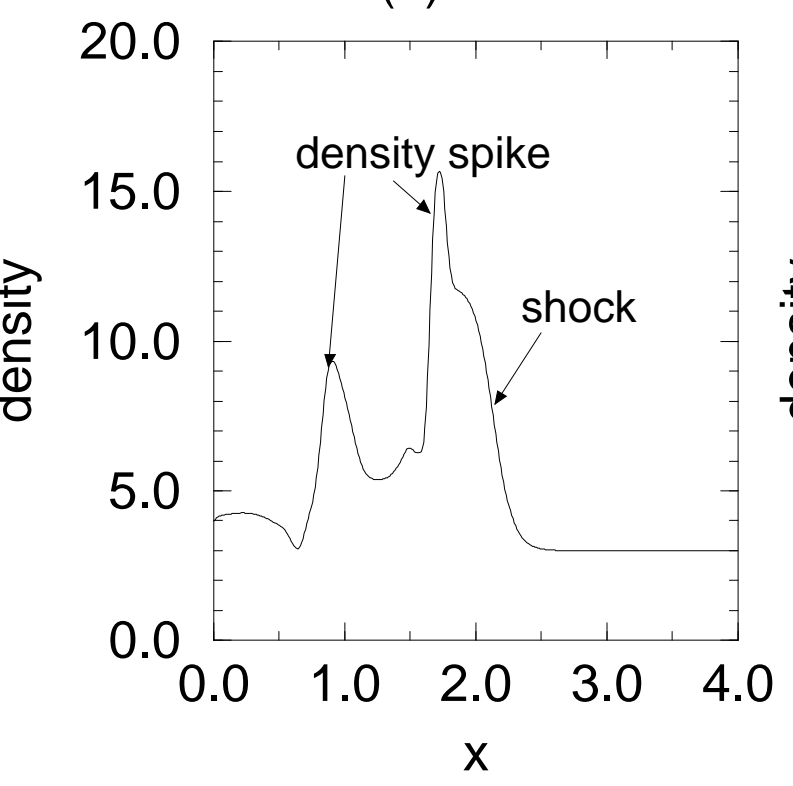

(c) $t=0.3$

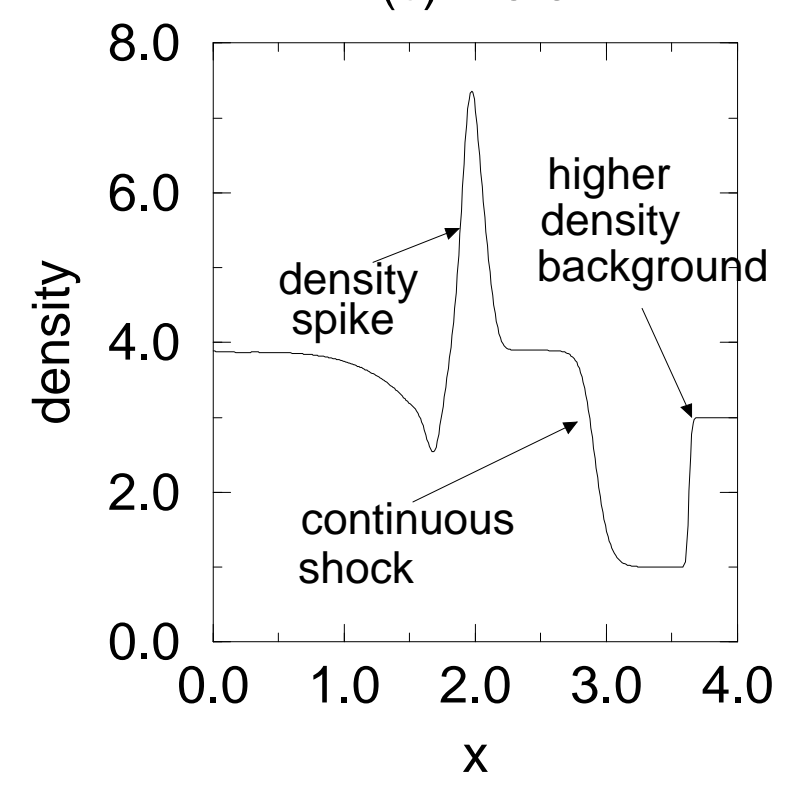

(f) $t=0.6$

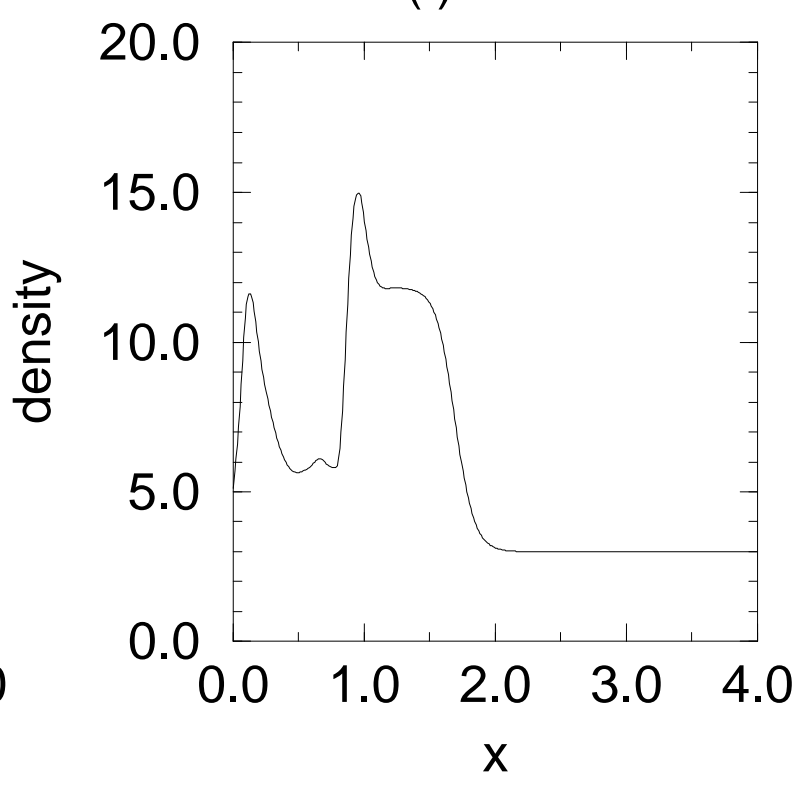



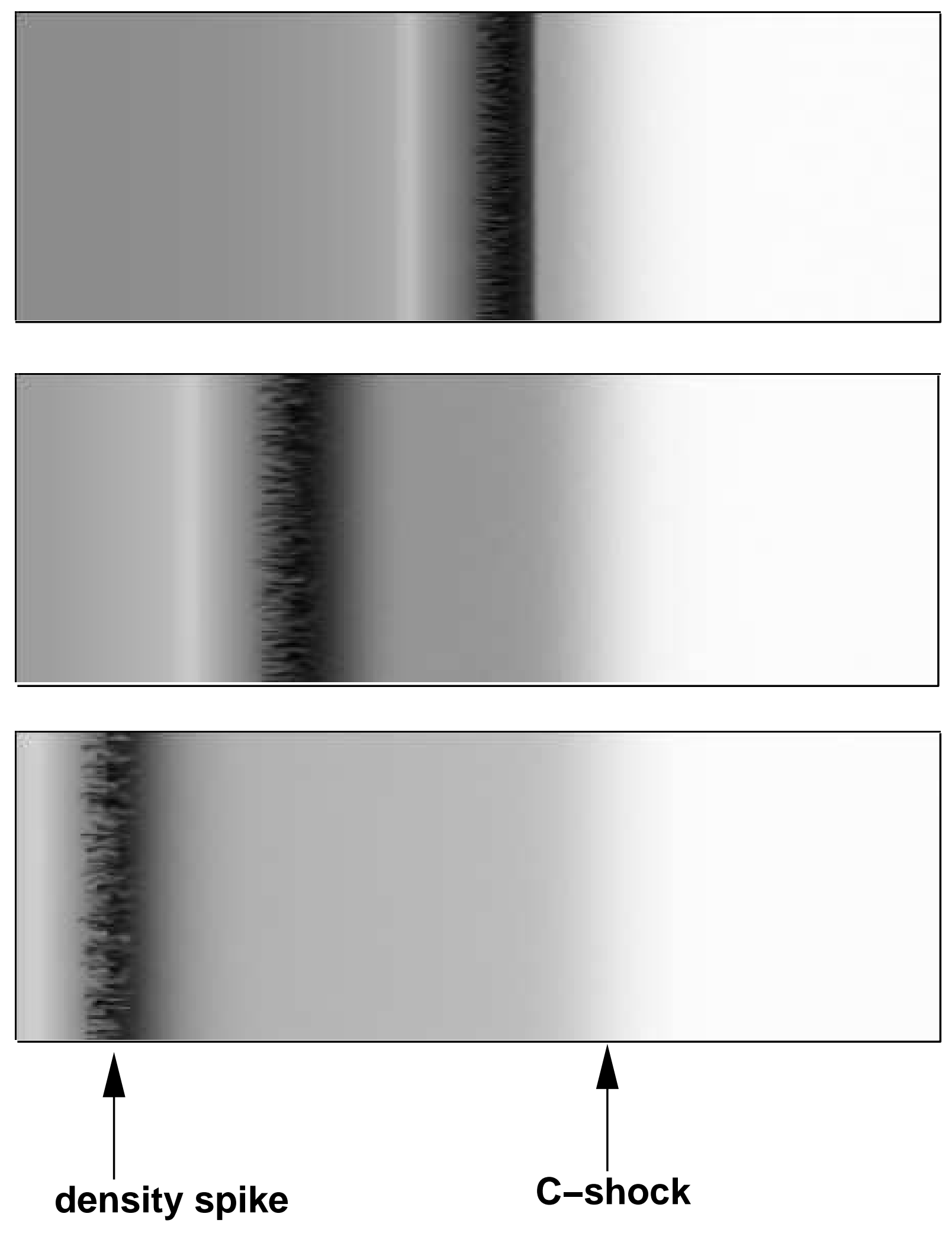


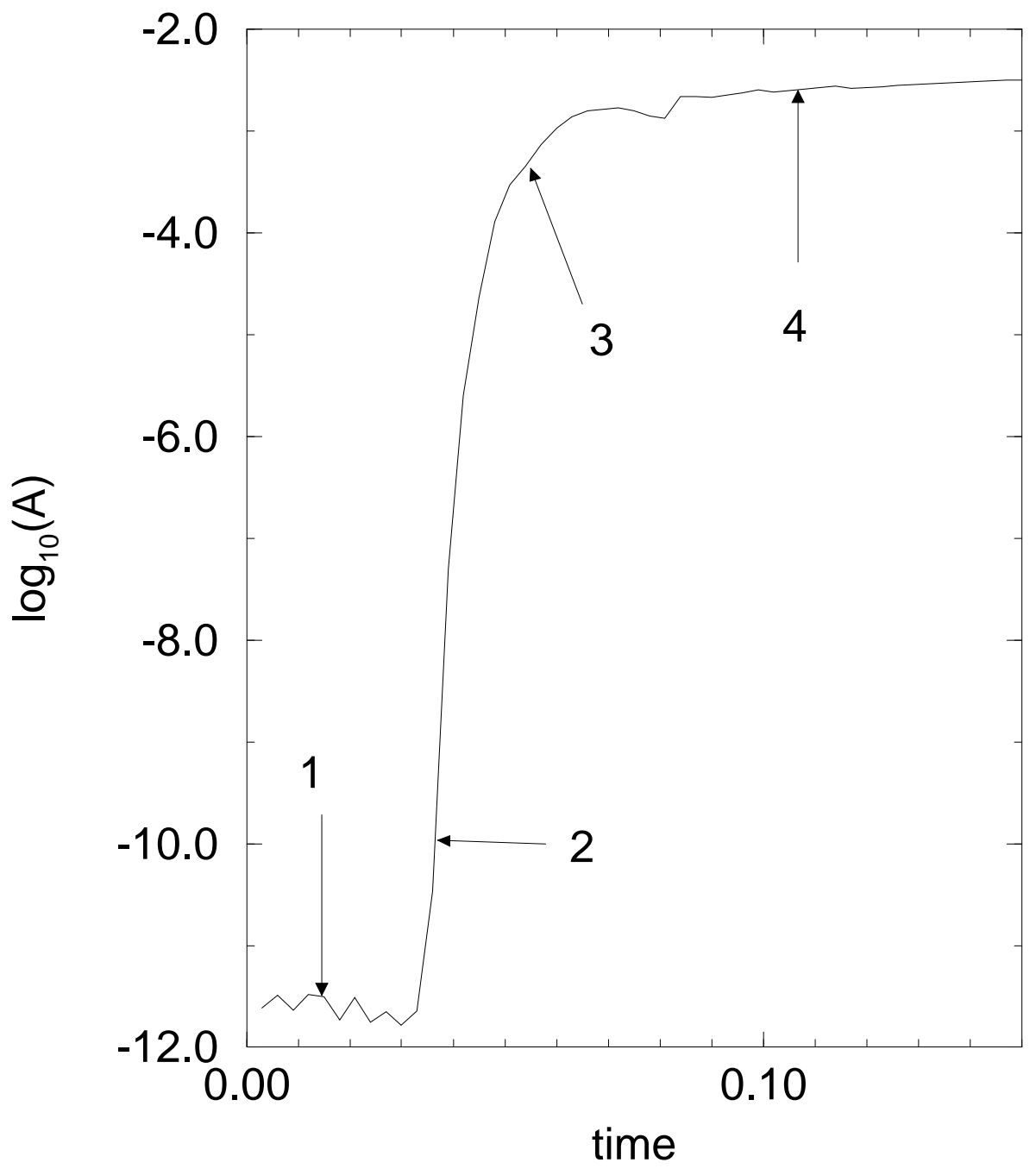




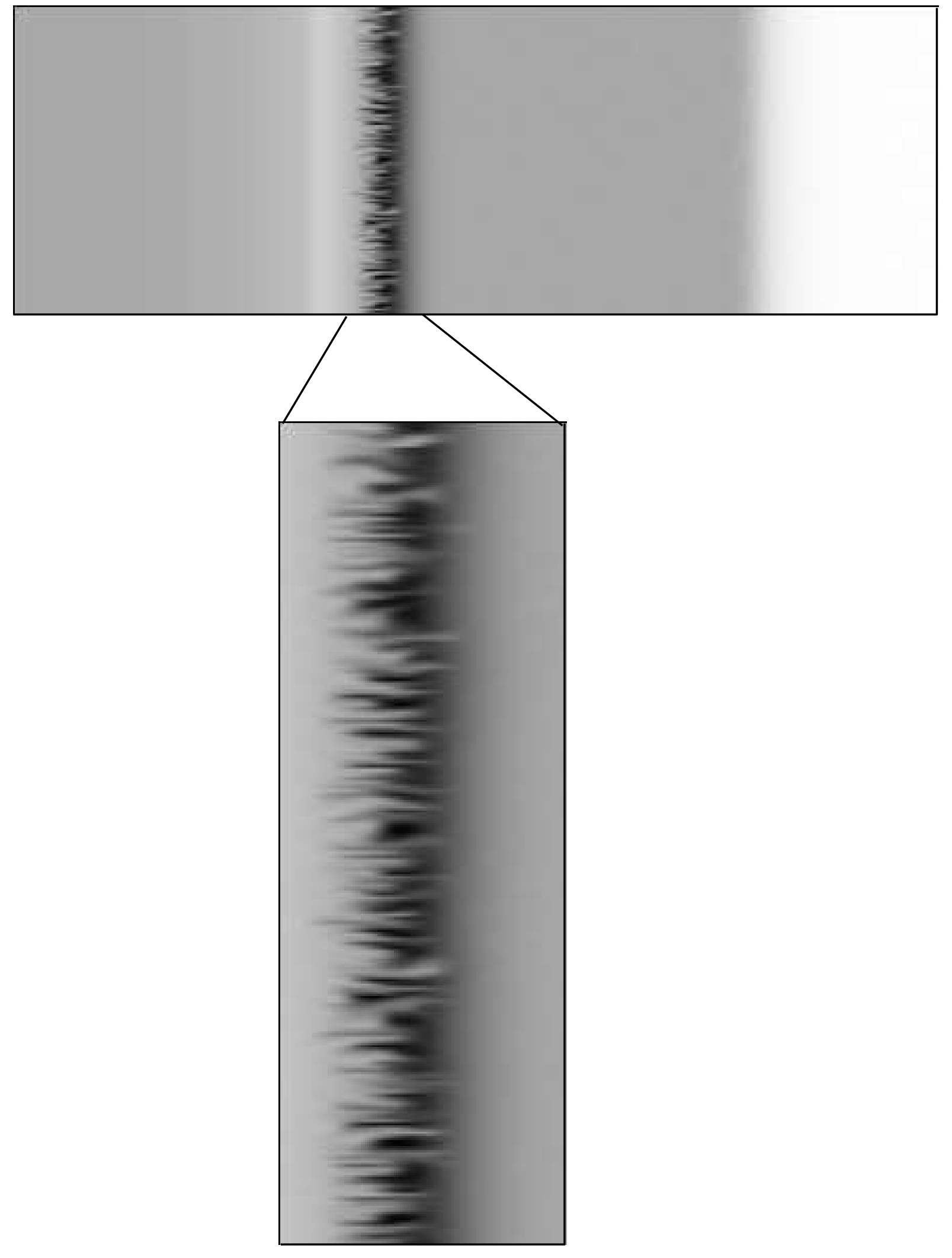

